\title{
Granular hypoplasticity with Cosserat effects
}

\author{
J. D. Goddard \\ Department of Mechanical and Aerospace Engineering \\ University of California, San Diego, \\ 9500 Gilman Drive \\ La Jolla, CA 92093-0411 USA
}

\begin{abstract}
This paper provides an extension to Cosserat mechanics of a recently proposed version of hypoplasticity [1], and this extension is achieved economically by means of a novel complex-variable formulation of Cosserat theory.

The present work represents a compact synthesis and theoretical framework for both non-polar and polar hypoplasticity, and it encompasses various special cases considered in the literature, as discussed in the recent monograph by Tejchman [2].

The current approach offers a perspective on granular dilatancy, elastoplastic yield, and energy dissipation which differs from the standard hypoplasticity and which serves to establish a connection to classical incremental plasticity. In contrast to the classical theory, the present approach, based entirely on the concept of pseudo-linear forms, admits but does require elastoplastic potentials to describe plastic flow. When such potentials are assumed, it is shown that they can be related to the plastic moduli of the present formulation.

It is also shown that hypoplasticity allows for a distinction between active and passive internal variables, with the latter serving to define parameters. Finally, the known forms for linear isotropic Cosserat elasticity are employed to represent isotropic hypoplasticity, and the resulting formulae appear to encompass several empiricisms found in the literature.
\end{abstract}

Keywords: micropolar, Cosserat continuum, parametric hypoplasticity, stiff elastoplasticity, couple stress, dilatancy PACS: $81.05 . \mathrm{Rm}, 83.80 . \mathrm{Fg}$

\section{INTRODUCTION}

Under the rubric of parametric hypoplasticity, the author has proposed in a recent work [1], hereinafter denoted by "Ref. I", a generalized version of conventional hypoplasticity [3] for assemblies of non-cohesive, nearly rigid particles. Starting from the notion of strictly dissipative materials and proceeding through incremental elastoplasticity, it provides a set of rate-independent ODEs, representing a minimal mathematical framework for the continuum modeling of the elastoplasticity of granular media, including:

1. elastoplastic yield with small-strain elastic response,

2. pressure-sensitive dilatant plasticity,

3. history-dependence, including the development of anisotropy based on evolutionary granular "texture" or "fabric", and

4. non-negative dissipation, based on stiff elastoplasticity.

Following a brief summary of the previous work, we consider the incorporation of Cosserat effects. As mentioned in the Abstract, many special cases of the Cosserat model are to be found in the literature [4, 5, 6, 7, 8, 9, 10, 11]. Much of the history and many theoretical concepts are covered in the recent monograph of Tejchman [2], which is focused on numerical simulations of soil mechanics based on standard hypoplastic modeling. As evident from this monograph, the exhaustive studies of Bauer and co-workers, e.g. [12], provide a compelling mathematical approach to the largely empirical "critical-state" soil mechanics.

As is also evident from the previous works, the Cosserat model provides one of the simplest of the numerous multipolar continuum models, which we recall serve to regularize simple-continuum models, e.g. in granular shear bands, by the introduction of additional kinematic gradients involving material length scales.

The present article aims to provide a systematic and concise treatment of the underlying theory. Although space does not allow for a comprehensive discussion of the various constitutive models presented in the above-cited literature, the present treatment may suggest a more systematic approach to such modeling.

The style of the exposition reflects the opinion, inspired by several treatises [13, 14, 3], that a compact formulation in direct tensor notation serves to elucidate otherwise complex expressions couched in tensor components, notwith- 
standing the computational merits of the latter. To those who share this view, the present work may serve as a useful overview of the literature.

\section{Nonpolar hypoplasticity}

It is worthwhile summarizing briefly the approach to hypoplasticity that is presented in more detail in Ref. I. With a view to thermodynamically admissibility, and in contrast to the standard expositions [15, 3], we proceed from the special case of a strictly dissipative material, in which the local Cauchy stress $\mathbf{T}(t)$ is given, modulo work-free reactions to any internal constraints, by the linear form in the local deformation rate $\mathbf{D}(t)$ :

$$
\mathbf{T}(t)=\boldsymbol{\eta}: \mathbf{D}(t) \text {, i.e. } T_{i j}(t)=\eta_{i j k l} D^{k l}(t)
$$

where $\boldsymbol{\eta}$ is a positive-definite viscosity depending on $\mathbf{D}(t)$ or $\mathbf{T}(t)$ and local deformation history. With the modification described below, (1) describes rigid plasticity, and, within the framework of parametric hypoplasticity, the history dependence is described by a set $\mathcal{A}$ of internal variables which satisfy rate-independent ${ }^{1}$ ODEs.

We generally adopt the notation of Ref. I, with vectors and second-rank tensors denoted, respectively, by bold lowercase Roman and uppercase Roman or Greek symbols, and with fourth-rank tensors, regarded as linear operators on second-rank tensors, denoted by bold lowercase Greek, $\boldsymbol{\alpha}, \boldsymbol{\beta}, \ldots$. As an exception, lowercase Greek is used for vectors representing Cosserat rotations, and Blackboard Bold is employed for complex variables, with real and imaginary parts denoted, respectively, by $\mathfrak{R}, \mathfrak{J}$, and overbars denoting complex conjugates.

The respective idemfactors are denoted by $\mathbf{I}$, with components $\delta_{i j}$, and by $\boldsymbol{\delta}$, with components $\delta_{i j k l}=\delta_{i k} \delta_{j l}$, and we employ standard tensor notation and summation convention for components on arbitrary curvilinear coordinates, with colons denoting linear operations on tensors represented by ordered pair-wise contraction on the trailing indices of prefactors with leading indices of postfactors.

Primes denote deviators:

$$
\mathbf{A}^{\prime}:=\mathbf{A}-\frac{1}{3} \mathbf{I}(\mathbf{I}: \mathbf{A}), \ldots \boldsymbol{\alpha}^{\prime}:=\boldsymbol{\alpha}-\frac{1}{3} \mathbf{I}(\mathbf{I}: \boldsymbol{\alpha}), \ldots
$$

and superposed carat denotes the versor (or director) of real second rank tensors ${ }^{2}$ :

$$
\hat{\mathbf{A}} \stackrel{\text { def }}{=} \frac{\mathbf{A}}{|\mathbf{A}|} \text {, where }|\mathbf{A}| \stackrel{\text { def }}{=}\left(\mathbf{A}: \mathbf{A}^{\mathrm{T}}\right)^{1 / 2} \equiv A_{i j} A^{i j},
$$

while superscripts $\mathrm{T}$ and $*$ denotes respective transpose or tensorial duals, with $A_{i j}^{\mathrm{T}} \stackrel{\text { def }}{=} \bar{A}_{j i}$ and $\alpha_{i j k l}^{*} \stackrel{\text { def }}{=} \bar{\alpha}_{k l i j}$, etc.

We denote various 4 th-rank tensor moduli by the symbol $\boldsymbol{\mu}$ and the corresponding compliances by $\boldsymbol{\kappa}=\boldsymbol{\mu}^{-1}$, such that, whenever these represent invertible linear transformations on the space of second-rank tensors,

$$
\left.\boldsymbol{\kappa}: \boldsymbol{\mu}=\boldsymbol{\delta} \text {, (i.e. } \kappa_{i j k l} \mu_{. . m n}^{k l}=\delta_{i j m n}\right) \text {, }
$$

The symbols $\left({ }^{\circ}\right) \stackrel{\text { def }}{=} d() / d t$ denote material time-derivatives defined by (18) below.

The relation (1) provides a theoretical framework for the rheology of idealized rigid-particle suspensions in viscous fluids [1], and a model of rate-independent rigid plasticity is obtained on replacing $\boldsymbol{\eta}$ by $\boldsymbol{\mu}_{\mathrm{p}} /|\mathbf{D}|$, where $\boldsymbol{\mu}_{\mathrm{P}}=\boldsymbol{\mu}_{\mathrm{p}}(\mathcal{A}, \hat{\mathbf{D}})$ defines a positive-definite plastic modulus. A dissipative flow rule and yield condition are then given, respectively, by

$$
\hat{\mathbf{D}}=\boldsymbol{\kappa}_{\mathbf{P}}: \mathbf{T}, \text { and } Y=\|\mathbf{T}\|_{\zeta}^{2} \stackrel{\text { def }}{=} \mathbf{T}: \zeta: \mathbf{T}=1 \text {, where } \zeta=\boldsymbol{\kappa}_{\mathbf{P}}^{*}: \boldsymbol{\kappa}_{\mathbf{P}}
$$

with $Y<1$ defining rigid states. In the standard granular model, $\boldsymbol{\kappa}_{\mathbf{P}}=p \boldsymbol{\kappa}_{\mathbf{C}}$, where $p=-\operatorname{tr}(\mathbf{T}) / 3>0$, and $\boldsymbol{\kappa}_{\mathrm{C}}$ is a nondimensional Coulomb compliance.

By the extension of an analysis relating elastic moduli and elastic potentials in Ref. I, Appendix A establishes a relation between plastic moduli and plastic potentials, on the presumption that the latter exist.

\footnotetext{
1 As an historical note, it is recalled that rate-independence reflects the absence of a characteristic material time scale, as pointed out in an article on particulate mechanics[16] that cites the work of Pipkin and Rivlin [17] but overlooks a germane work by Gudehus [18] on granular materials.

2 to be distinguished from the use of carats in certain works [8] to denote stress normalized by isotropic pressure
} 
An accounting for elastic effects, based on the elastic-plastic decomposition $\mathbf{D}=\mathbf{D}_{\mathbf{E}}+\mathbf{D}_{\mathbf{P}}$, where $\mathbf{D}_{\mathbf{P}}$ satisfies (5), and deformation rate $\mathbf{D}_{\mathbf{E}}$ is given in terms of stress rate by an incremental elastic modulus $\boldsymbol{\mu}_{\mathrm{E}}$, leads to the hypoplastic form [1]

$$
\stackrel{\circ}{\mathbf{T}}=\boldsymbol{\mu}_{\mathrm{H}}: \mathbf{D}-|\mathbf{D}| \mathbf{N} \text {, with } \boldsymbol{\mu}_{\mathrm{H}} \equiv \boldsymbol{\mu}_{\mathrm{E}} \text {, and } \mathbf{N}=\beta \boldsymbol{\mu}_{\mathrm{E}}: \boldsymbol{\kappa}_{\mathrm{P}}: \mathbf{T} \text {, }
$$

where $\stackrel{\circ}{\mathbf{T}}$ represents an objective (e.g. Jaumann) rate, and $\beta$ an "inelastic-clock" function (denoted by $\vartheta$ in Ref. I) that vanishes inside the yield surface, e.g., in terms of a particular Heaviside function:

$$
\beta=H(Y-1), \text { with } H(0) \stackrel{\text { def }}{=} H(0+)=1
$$

The conventional hypoplastic model $[15,3,2]$ takes $\beta \equiv 1$, with no distinction between plastic and total deformation rates, and assumes $\boldsymbol{\mu}_{\mathrm{H}}$ and $\mathbf{N}$ to be functions of $\mathbf{T}(t)$, without symmetry or positivity restrictions on $\boldsymbol{\mu}_{\mathrm{H}}$. While the model does not identify a yield surface per se, the "stationary state" where stress rate vanishes represents the dissipative yield surface identified in the present work, subject to the interpretation of $\boldsymbol{\mu}_{\mathrm{H}}$ and $\mathbf{N}$ in (6). Furthermore, and contrary to occasional remarks in the literature, hypoplasticity does allow for elastic response, provided that term $\mathbf{N}$ become negligible for small $|\mathbf{T}|$, which is suggested immediately by the form of (6).

Without pursuing the details [1] here, we note that the interpretation of dilatancy as an internal constraint requires that the expression for $\mathbf{N}$ in (6) be replaced by

$$
\mathbf{N}=\frac{\beta}{\sqrt{1+\alpha^{2} / 3}} \boldsymbol{\mu}_{\mathbf{E}}:\left(\boldsymbol{\kappa}_{\mathbf{P}}^{\prime}: \mathbf{T}+\frac{1}{3} \alpha \mathbf{I}\right),
$$

where $\boldsymbol{\kappa}_{\mathbf{P}}^{\prime}$ is a deviatoric plastic compliance and $\alpha$ is a coefficient of dilatancy [1]. Furthermore, in the standard granular model, one has $\boldsymbol{\kappa}_{\mathrm{P}}^{\prime}=\boldsymbol{\kappa}_{\mathrm{C}}^{\prime} / p$, where $p$ is confining pressure and $\boldsymbol{\kappa}_{\mathrm{C}}^{\prime}$ a nondimensional Coulomb compliance that is independent of $p$.

As a final observation, we note that (6) is a special case of the rate-independent pseudo-linear ${ }^{3}$ form:

$$
\stackrel{\circ}{\mathbf{T}}=\boldsymbol{\pi}(\mathbf{T}, \hat{\mathbf{D}}): \mathbf{D}, \text { with } \boldsymbol{\pi} \stackrel{\text { def }}{=} \boldsymbol{\mu}_{\mathbf{H}}-\mathbf{N}_{\mathbf{H}} \otimes \hat{\mathbf{D}} \text {, i.e. } \pi_{i j k l}=\mu_{i j k l}-N_{i j} \hat{D}_{k l},
$$

which obviously reduces elastoplasticity to a set of rate-independent ODEs with $\mathbf{D}$ as control variable.

Unfortunately, there is no guarantee of thermodynamic admissibility of the general form (9), which, for example, may fail to exhibit non-negative work on cycles. In contrast, the interpretation (6) in terms of a physically-motivated incremental elastoplasticity leads to a weak form of Il'yushin's postulate discussed in Ref. $\mathrm{I}^{4}$, and this provides yet another motivation for the use of evolutionary internal variables.

\section{Parametric Hypoplasticity}

This more general version of hypoplasticity, anticipated in early works on the subject [19, 15] has been computationally implemented in numerous works by Tejchman, Bauer and co-workers [20, 7, 12, 8, 21, 22] for polar as well as non-polar media. In the non-polar form, these models involve internal variables whose history dependence is determined by ODEs having the pseudo-linear form:

$$
\stackrel{\circ}{\mathcal{T}}=\mathcal{P}(\mathcal{T}, \hat{\mathbf{D}})[\mathbf{D}], \text { with } \mathcal{T}=\{\mathbf{T}, \mathcal{A}\}
$$

where $\mathcal{P}[\mathbf{D}]$ is linear in $\mathbf{D}$, and $\mathcal{A}$ denotes the set of internal variables. While the unqualified term "hypoplasticity" appears to enjoy general usage in the current literature, the adjective parametric is employed here to emphasize the special nature of parameters regarded as internal variables. As discussed below, the term parameter refers to any internal variable whose power (work-rate) can be derived from the kinematics represented by D. This is made more precise below, after consideration of some typical examples of parameters.

\footnotetext{
${ }^{3}$ As $\mathbf{Y}=\boldsymbol{\pi}(\hat{\mathbf{X}}): \mathbf{X}=\Pi(\hat{\mathbf{X}})|\mathbf{X}|$, i.e. $Y_{i j}=\pi_{i j}{ }^{k l} X_{k l}=\Pi_{i j}(\hat{\mathbf{X}})|\mathbf{X}|$, reflects the degree-one homogeneity of cones, other terminology might be suggested.

4 which also overlooks the older work of Gudehus [18], who considers the relevance of Il'yushin's postulate to granular plasticity.
} 
In the simplest example [12], $\mathcal{A}$ is given solely by the void ratio $e$, determined through plastic volume change by:

$$
\stackrel{\circ}{e} \equiv \dot{e}=(1+e) \operatorname{tr}\left(\mathbf{D}_{\mathbf{P}}\right)=\frac{(1+e) \alpha \beta}{\sqrt{1+\alpha^{2} / 3}}|\mathbf{D}|
$$

Then, with $\boldsymbol{\mu}_{\mathrm{E}}, \boldsymbol{\kappa}_{\mathbf{P}}^{\prime}, \alpha$ in (6) depending on $\mathcal{X},(10)$ is represented by (6), (7) and (11). We recall that the standard hypoplastic model [12] without dilatancy constraint assumes $\beta=1, \alpha=\sqrt{3 / 2}$.

A discussion of various limiting forms of this model is given in Ref. I, including "stiff" elastoplasticity, where

$$
\epsilon=\left\|\boldsymbol{\kappa}_{\mathrm{E}}\right\| /\left\|\boldsymbol{\kappa}_{\mathrm{P}}\right\|=p\left\|\boldsymbol{\kappa}_{\mathbf{E}}\right\| /\left\|\boldsymbol{\kappa}_{\mathrm{C}}\right\|<<1, \text { with } \alpha=O\left(\epsilon^{-1}\right)
$$

In this limit, (6) reduces to a stiff ODE, with $\mathbf{D} \approx \mathbf{D}_{\mathbf{P}}$, except on small strain scales $O(\epsilon)$ near points of elastic loading or unloading. As pointed out in Ref. I, the relation (12) is particularly apt for stiff geomaterials such as sand.

Another candidate for scalar parameter is the accumulated plastic shear strain $\gamma_{\mathbf{P}}$ common to numerous plasticity theories, which satisfies the further ODE:

$$
\dot{\gamma}_{\mathbf{P}} \stackrel{\text { def }}{=}\left|\mathbf{D}_{\mathbf{P}}^{\prime}\right|=\frac{\beta}{\sqrt{1+\alpha^{2} / 3}}|\mathbf{D}|
$$

Since $\alpha$ and $\beta$ may generally depend on both $e$ and $\gamma_{\mathbf{P}}$, both ODEs (11) and (13) are needed.

For isotropic materials, the moduli and compliances can be represented as isotropic polynomials in $\mathbf{T}$ or $\widehat{\mathbf{D}}^{\prime}$, with scalar coefficients depending on their joint isotropic scalar invariants and on a set of scalar parameters such as $e$ and $\gamma_{\mathbf{P}}$ cited above. However, in order to describe strain-induced anisotropy in a material which is isotropic in some virgin state, one needs additional tensorial variables [18], such as a 2nd-rank fabric ${ }^{5}$ tensor $\mathbf{A}$. In this case, a general form of (10) is given by the higher-order ODE for parametric evolution $[16,1]$ obtained by taking $\mathcal{A}=\left\{\mathbf{A}_{1}, \mathbf{A}_{2}, \ldots, \mathbf{A}_{n}\right\}$, $\mathbf{A}_{1} \equiv \mathbf{A}$, with

$$
\begin{gathered}
{\stackrel{\circ}{\mathbf{A}_{k}}}_{k}=\beta|\mathbf{D}| \mathbf{A}_{k+1}, \text { for } k=1, \ldots, n-1, \\
\stackrel{\circ}{\mathbf{A}}_{n}=\boldsymbol{\pi}(\mathcal{X}, \hat{\mathbf{D}}): \mathbf{D}, \quad \mathcal{X}=\left\{\mathbf{T}, \gamma_{\mathbf{p}}, e, \mathcal{A}\right\},
\end{gathered}
$$

together with (11) and (13), where $\boldsymbol{\pi}$ represents a pseudo-linear form like that in (9), and all coefficients $\alpha, \beta, \ldots$ depend on $\mathcal{X}$, with $\pi$ given generally as a tensor polynomial in $\mathbf{T}, \hat{\mathbf{D}}, \mathbf{A}$. The associated anisotropy is to be distinguished from inherent or "initial" anisotropy [22] associated with certain virgin states of the material.

Recent work on concentrated fluid-particle suspensions discussed in Ref. I suggests that a low-order model, with $n \leq 2$ and with a low-order tensor polynomial in $\mathbf{A}$, may suffice to describe induced anisotropy in simple shear. It remains to be seen whether a similar simplification applies to granular media subject to more complex deformation histories, but recent numerical simulations [25] are encouraging.

\section{Passive vs. active variables}

Underlying the continuum-mechanical principle of virtual work, internal variables represent configurational degree of freedom endowed with specific mechanical power and work-conjugate stress, the latter representing the dual space to the configurational tangent space. However, within the framework of parametric hypoplasticity, parameters represent a special class of internal variables whose kinematics are given as pseudo-linear forms in $\mathbf{D}$, implying that their stress and stress power are not independent of the usual mechanical quantities.

For example in the case of void ratio $e$, the power is given by $p \dot{e} /(1+e)$, where $p=-\operatorname{tr}(\mathbf{T}) / 3$, whereas for accumulated plastic shear $\gamma_{\mathbf{P}}$ defined by $(13)^{6}$, it is easy to show that the power is given by $\tau_{\mathrm{P}} \dot{\gamma}_{\mathrm{P}}$, with $\tau_{\mathrm{P}}=\mathbf{T}: \boldsymbol{\kappa}_{\mathrm{P}}: \mathbf{T}$.

In the case of fabric, with conjugate stress $\mathbf{S}_{\mathbf{A}}$,

$$
\stackrel{\circ}{\mathbf{A}}=\boldsymbol{\pi}: \mathbf{D} \Rightarrow \mathbf{S}_{\mathbf{A}}: \stackrel{\circ}{\mathbf{A}}=\mathbf{T}_{\mathbf{A}}: \mathbf{D}, \text { where } \mathbf{T}_{\mathbf{A}}=\mathbf{S}_{\mathbf{A}}: \boldsymbol{\pi}(\mathbf{T}, \mathcal{A}, \hat{\mathbf{D}})
$$

\footnotetext{
5 "Texture" is often employed in the metal-plasticity literature to denote a similar concept, a term dating at least as far back as the early work of Hill [23]. That work does not provide a complete description of strain-induced evolution of anisotropy, and a cursory survey of subsequent literature suggests that, with notable exceptions [24], attempts are rarely made to relate micro-level to continuum-level descriptions of texture evolution.

${ }^{6}$ Conversely, given any norm $\tau=\|\mathbf{T}\|$, one can define $\dot{\gamma}_{\mathbf{P}}=\hat{\mathbf{D}}: \boldsymbol{\mu}_{\mathbf{P}}: \hat{\mathbf{D}} / \tau$, which appears equivalent to Hill's [26] duality based on plastic potentials.
} 
In this sense, the nominal hypoplastic parameters may be treated as passive internal variables, enslaved to D. On the other hand, the Cosserat rotations considered next do not qualify as parameters, since they involve independent kinematics, with conjugate stresses and work effects that are not derivable from $\{\mathbf{T}, \mathcal{A}, \hat{\mathbf{D}}\}$.

\section{COSSERAT EFFECTS}

The following is a highly condensed restatement of Cosserat mechanics, which is treated at much greater length in many previous works $[27,13,28,29,30,4,5]$. From the conventional continuum-mechanical viewpoint, a Cosserat continuum $^{7}$ is defined by a differentiable map assigning spatial position $\mathbf{x}\left(\mathbf{x}^{\circ}, t\right)$ and microstructural rotation $\mathbf{P}\left(\mathbf{x}^{\circ}, t\right)$ to each material particle, with $\mathbf{x}=\mathbf{x}^{\circ}, \mathbf{P}=\mathbf{I}$ in a given reference configuration, where $\mathbf{P} \in \mathbf{S O}(3)$ denotes a real, proper orthogonal tensor (cf. [34]).

\section{Kinematics - rotation and spin}

Following Mindlin [28, 29], in a slight departure from several previous works [30, 8], we employ a rotation relative to the material rather than to a fixed spatial frame. In either case, we can express the kinematics concisely in terms of the map $\mathbb{R}^{3} \rightarrow \mathbb{R}^{3} \times \mathbf{S O}(3)$ given by

$$
\begin{aligned}
& \mathbf{x}^{\circ} \rightarrow\{\mathbf{x}, \boldsymbol{\theta}\}, \text { where } \boldsymbol{\theta}=-\frac{\mathbf{1}}{\mathbf{2}} \boldsymbol{\epsilon}: \boldsymbol{\Theta} \text {, and } \boldsymbol{\Theta}=-\boldsymbol{\epsilon} \cdot \boldsymbol{\theta}, \\
& \text { i.e. } \theta_{i}=-\frac{1}{2} \epsilon_{i j k} \Theta^{j k} \text {, and } \Theta_{i j}=-\epsilon_{i j k} \theta^{k},
\end{aligned}
$$

and the Cayley-Gibbs-Rodrigues relation [13, 35, 36, 37, 38, 39]

$$
\mathbf{P}=\exp \Theta=\mathbf{I}+\left(\frac{\sin \vartheta}{\vartheta}\right) \Theta+\left(\frac{1-\cos \vartheta}{\vartheta^{2}}\right) \Theta^{2}, \vartheta=\left\{-\operatorname{tr}\left(\Theta^{2}\right) / 2\right\}^{1 / 2}
$$

Here $\hat{\boldsymbol{\theta}}=\boldsymbol{\theta} /|\boldsymbol{\theta}|$ represents the axis of rotation and $\vartheta=|\boldsymbol{\theta}|=\left(\theta^{i} \theta_{i}\right)^{1 / 2}$ the angle of rotation about the axis.

If $\mathbf{P}$ is taken as primary variable, the skew-symmetric tensor $\boldsymbol{\Theta}=\log \mathbf{P} \in \mathfrak{s}$ (3) (Lie algebra) represents an inverse of the map $\mathfrak{s}$ (3) $\rightarrow \mathbf{S O}$ (3) (Lie group), and it can be defined uniquely and computed by various methods [40]. Alternatively, and more conveniently, we may regard $\boldsymbol{\theta}$ as the primary variable, with (16) defining the associated map or Cosserat placement $\mathbb{R}^{3} \rightarrow \mathbb{R}^{6}$.

To connect the vector of the logarithmic spin $\mathbf{\Omega}=d \boldsymbol{\Theta} / d t$ to that of the instantaneous spin $\mathbf{\Upsilon}=(d \mathbf{P} / d t) \mathbf{P}^{\mathrm{T}}$, where

$$
\frac{d}{d t} \stackrel{\text { def }}{=}\left(\frac{\partial}{\partial t}\right)_{\mathbf{x}^{\circ}},
$$

we recall the rather remarkable result of Kafadar and Eringen [30, Eqs.(2)-(9)], which can be expressed as:

$$
\begin{aligned}
& \qquad \boldsymbol{\nu} \stackrel{\text { def }}{=}-\frac{1}{2} \boldsymbol{\epsilon}: \boldsymbol{\Upsilon}=\boldsymbol{\Gamma} \boldsymbol{\omega}, \text { where } \boldsymbol{\omega}=-\frac{1}{2} \boldsymbol{\epsilon}: \boldsymbol{\Omega}, \\
& \text { and } \\
& \qquad \boldsymbol{\Gamma}=\mathbf{I}+\left(\frac{1-\cos \vartheta}{\vartheta^{2}}\right) \Theta+\left(\frac{\vartheta-\sin \vartheta}{\vartheta^{3}}\right) \Theta^{2}, \text { with } \boldsymbol{\Gamma}^{-1}=\mathbf{I}-\frac{1}{2} \Theta+\frac{1}{\vartheta^{2}}\left(1-\frac{\vartheta}{2} \cot \frac{\vartheta}{2}\right) \Theta^{2}
\end{aligned}
$$

Either definition of spin is acceptable, and this relation makes it easy to connect their work-conjugate stresses ${ }^{8}$.

It should be noted that (19) has also been derived by Shuster [42, Eqs. (276)-279] ${ }^{9}$, and that Iserle et al. [37, Eqs. B.10-B.11] present it as the differential of the Lie-group exponential, cf. [36, Eqs. 17-19]. It can also be obtained from the representation of rotations by quaternions [43].

\footnotetext{
${ }^{7}$ More general continua [31] are obtained by assigning additional kinematic variables to material points, the simplest example being Eringen's microstretch continuum [30] involving dilatation as well as rotation. Although sometimes proposed as a model of granular dilatancy [32, 33], the physical significance is not clear. In the typical granular system, the dilatation of individual grains is small compared to that of void space, which is already represented by a simple continuum with variable density or void ratio $e$.

${ }^{8}$ Owing no doubt to the group character of rotations, this relation is much simpler than that connecting the conjugate stress for logarithmic (Hencky) strain to Cauchy stress, as illustrated by the work Xiao et al. [41].

${ }^{9}$ pointed out to me by Professor Reuven Segev
} 


\section{Complex-variable representation}

Starting from (16), one can describe Cosserat kinematics by means of a complex placement

$$
\mathfrak{x}\left(\mathbf{x}^{\circ}\right)=\mathbf{x}+\imath \boldsymbol{\theta}
$$

leading to complex displacement and velocity field defined, respectively, by

$$
u=\mathbf{u}+\imath \boldsymbol{\theta}, \text { with } \mathbf{u}=\mathbf{x}-\mathbf{x}^{\circ} \text { and } \vee=\frac{d u}{d t}=\mathbf{v}+\imath \boldsymbol{\omega}, \text { where } \mathbf{v}=\frac{d \mathbf{u}}{d t}, \text { and } \boldsymbol{\omega}=\frac{d \boldsymbol{\theta}}{d t},
$$

More physically relevant quantities, particularly for small-strain theories, would be obtained by replacing $\boldsymbol{\theta}$ with $\xi \boldsymbol{\theta}$, where $\xi$ is a material length scale, e.g. a particle diameter. However, in an abstract mathematical sense, the form (20) is equally valid, with the proviso that physically meaningful norms must involve a length scale, e.g.

$$
|\mathbf{u}|=\sqrt{|\mathbf{u}|^{2}+\xi^{2}|\boldsymbol{\theta}|^{2}}
$$

which of course differs from the usual complex-variable modulus.

As a generalization of the standard deformation gradient $\mathbf{F}$ [14], we have:

$$
\mathbb{F}=\partial \mathfrak{x} / \partial \mathbf{x}^{\circ}=\mathbf{F}+{ }_{l} \mathbf{G}, \text { where } \mathbf{F}=\partial \mathbf{x} / \partial \mathbf{x}^{\circ}, \mathbf{G}=\partial \boldsymbol{\theta} / \partial \mathbf{x}^{\circ}
$$

with corresponding rate ${ }^{10}$

$$
\frac{d \mathbb{F}}{d t}=\mathbb{L} \mathbf{F}, \text { with } \mathbb{L}=(\boldsymbol{\nabla} \vee)^{\mathrm{T}}=(\mathbf{L}+\imath \mathbf{K}), \mathbf{L}=(\boldsymbol{\nabla} \mathbf{v})^{\mathrm{T}}, \mathbf{K}=(\boldsymbol{\nabla} \boldsymbol{\omega})^{\mathrm{T}},
$$

where the deformation gradient and Cosserat rotation satisfy the ODEs:

$$
\left.\frac{d \mathbf{F}}{d t}=\mathbf{L F}, \frac{d \boldsymbol{\Theta}}{d t}=\boldsymbol{\Omega}, \text { where } \boldsymbol{\Omega}=-\boldsymbol{\epsilon} \cdot \boldsymbol{\omega}, \text { (i.e. } \Omega_{i j}=-\epsilon_{i j k} w^{k}\right)
$$

We recall that $\mathbf{K}$ and its conjugate $\mathbf{M}$ are expressible, respectively, in terms of a third-rank wryness or curvature (cf. [29]) and double-stress.

The above relations yield the frame-indifferent description of Cosserat mechanics, with complex asymmetric deformation rate $\mathbb{D}$ and stress $\mathbb{T}$, and stress-power $\dot{w}$ defined, respectively, by

$$
\begin{aligned}
& \qquad \mathbb{D}=\mathbf{\Lambda}+\imath \mathbf{K} \text {, with } \boldsymbol{\Lambda}=\mathbf{D}+\mathbf{\Omega}, \mathbb{T}=\boldsymbol{\Sigma}+\imath \mathbf{M}, \\
& \text { and } \\
& \qquad \dot{w}=\mathfrak{R}\left(\mathbb{T}: \mathbb{D}^{*}\right)=\boldsymbol{\Sigma}: \boldsymbol{\Lambda}+\mathbf{M}: \mathbf{K}=\mathbf{T}: \mathbf{D}+\mathbf{S}: \mathbf{\Omega}+\mathbf{M}: \mathbf{K}=\mathbf{T}: \mathbf{D}+\mathbf{s} \cdot \boldsymbol{\omega}+\mathbf{M}: \mathbf{K},
\end{aligned}
$$

where

$$
\mathbf{T} \stackrel{\text { def }}{=} \operatorname{Sym} \Sigma, \mathbf{S} \stackrel{\text { def }}{=} \operatorname{Skw} \boldsymbol{\Sigma}, \mathbf{D} \stackrel{\text { def }}{=} \operatorname{Sym} \mathbf{L}, \mathbf{S}=-\frac{1}{2} \boldsymbol{\epsilon}: \mathbf{S}
$$

with Sym and Skw denoting symmetric and skew-symmetric parts, respectively. Any of the tensors $\mathbf{Z}=\mathbf{D}, \ldots, \mathbf{M}$, transforms as $\mathbf{Z}^{+}=\mathbf{Q Z Z} \mathbf{Q}^{T}$ under a change of frame $\mathbf{F}^{+}=\mathbf{Q F}$, where $\mathbf{Q}^{-1}=\mathbf{Q}^{\mathrm{T}}$. Note that one can substitute the spin $\Upsilon$ for $\boldsymbol{\Omega}$ by merely changing the definition of the conjugate stress $\mathbf{S}$.

Although we shall not make use of them in the present work, it is particularly noteworthy that the standard linear and angular momentum balances $[28,29,30]$ can now be put into a remarkably compact form:

$$
\boldsymbol{\nabla} \cdot \mathbb{T}^{\mathrm{T}}+\mathfrak{b}=\rho \mathbb{a}, \text { with, } \mathfrak{b}=\mathbf{b}+\imath(\mathbf{s}+\mathbf{c}) \text {, and } \mathbb{a}=\mathbf{v}+\imath \mathbf{\Xi} \boldsymbol{\omega},
$$

where $\mathbb{a}$ is complex acceleration, $\mathbf{b}$ and $\mathbf{c}$ represent external body-force and body-couple density, respectively, and $\boldsymbol{\Xi}$ is a rotational microinertia tensor, different from but related to that employed in [30]. Hence, insofar as the momemtum balance is concerned, Cosserat mechanics is merely complex Cauchy mechanics.

As indicated next, this same complex-variable formulation makes for a rather compact generalization of the preceding hypoplastic constitutive theory.

\footnotetext{
${ }^{10}$ The less conventional form $x=x\left(\mathfrak{x}^{\circ}, t\right)$, with $\mathbb{F} \stackrel{\text { def }}{=} \partial \mathfrak{x} / \partial \mathfrak{x}^{\circ}$, would represent classes of reference states involving different rotations $\boldsymbol{\theta}^{\circ}$ at each material point.
} 


\section{Elastoplastic forms}

With real and imaginary components denoted, respectively, by subscripts $\mathfrak{R}$ and $\mathfrak{I}$, then

$$
\mathbf{D}_{\mathfrak{R}}=\Lambda, \mathbf{D}_{\mathfrak{J}}=\mathbf{K}, \mathbf{T}_{\mathfrak{R}}=\boldsymbol{\Sigma}, \mathbf{T}_{\mathfrak{I}}=\mathbf{M}
$$

and complex 4th-rank moduli are defined by:

$$
\mu=\boldsymbol{\mu}_{\mathfrak{R}}+\imath \boldsymbol{\mu}_{\mathfrak{J}},
$$

with a similar form for complex compliances, where it is understood that regular boldface denotes real quantities. Then, any essentially real linear map $\mathfrak{M}$, i.e., a map restricted to linear combinations with real coefficients, of complex 2 nd-rank tensors $\mathbb{X}$ into complex 2 nd-rank tensors $\mathbb{Y}$, can be represented by two complex moduli $\mu_{i}=\boldsymbol{\mu}_{i \Re}+\imath \boldsymbol{\mu}_{i \mathfrak{J}}$, for $i=1,2$. Of the several possibilities, we choose

$$
\mathbb{Y}=\mathfrak{M}: \mathbb{X} \stackrel{\text { def }}{=} \mathfrak{R}\left(\mu_{1}: \overline{\mathbb{X}}\right)+\imath \mathfrak{R}\left(\mu_{2}: \overline{\mathbb{X}}\right)
$$

with matrix representation

$$
\left[\begin{array}{l}
\mathbf{Y}_{\mathfrak{R}} \\
\mathbf{Y}_{\mathfrak{I}}
\end{array}\right]=\left(\begin{array}{ll}
\boldsymbol{\mu}_{1 \mathfrak{R}} & \boldsymbol{\mu}_{1 \mathfrak{I}} \\
\boldsymbol{\mu}_{2 \mathfrak{R}} & \boldsymbol{\mu}_{2 \mathfrak{J}}
\end{array}\right):\left[\begin{array}{l}
\mathbf{X}_{\mathfrak{R}} \\
\mathbf{X}_{\mathfrak{J}}
\end{array}\right],
$$

By an extension of the preceding terminology, we refer to the restricted linear operator $\mathfrak{M}$ as a "modulus", and, by matrix inversion, one then obtains a"compliance" $\Omega=\mathfrak{M}^{-1}$. Quotation marks are employed here to emphasize that the terminology should, strictly speaking, be reserved for matrix representations like that in (31), which are necessary because of the well-known limitation on complex-variable representations ${ }^{11}$.

With $\mathbb{X}=\mathbb{D}=\mathbf{\Lambda}+\imath \mathbf{K},(31)$ defines a pseudo-linear kinematic form, with associated (pseudo-)quadratic form:

$$
w \stackrel{\text { def }}{=} \mathfrak{R}\left(\mathbb{D}^{*}: \mathfrak{M}: \mathbb{D}\right)=\boldsymbol{\Lambda}^{\mathrm{T}}: \boldsymbol{\mu}_{1 \mathfrak{R}}: \boldsymbol{\Lambda}+\boldsymbol{\Lambda}^{\mathrm{T}}:\left(\boldsymbol{\mu}_{1 \mathfrak{J}}+\boldsymbol{\mu}_{2 \mathfrak{R}}^{*}\right): \mathbf{K}+\mathbf{K}^{\mathrm{T}}: \boldsymbol{\mu}_{2 \mathfrak{J}}: \mathbf{K}
$$

The requirement that $\mathfrak{M}$ be self-adjoint implies symmetry of the matrix in (31), with the consequence that

$$
\mu_{1 \mathfrak{J}}=\boldsymbol{\mu}_{2 \mathfrak{R}}^{*}, \boldsymbol{\mu}_{1 \mathfrak{R}}=\boldsymbol{\mu}_{1 \mathfrak{R}}^{*}, \boldsymbol{\mu}_{2 \mathfrak{J}}=\boldsymbol{\mu}_{2 \mathfrak{J}}^{*},
$$

and with corresponding reduction of the quadratic form (32). The further condition of positivity obviously requires inter alia the positivity of $\boldsymbol{\mu}_{1 \Re}$ and $\boldsymbol{\mu}_{2 \mathfrak{J}}$.

For the sake of completeness, Appendix B presents the isotropic form of (31)-(32), for which both $\boldsymbol{\mu}_{1 \mathfrak{J}}$ and $\boldsymbol{\mu}_{2 \mathfrak{R}}$ vanish, leaving only two moduli $\boldsymbol{\mu}_{1 \mathfrak{R}}=\boldsymbol{\mu}_{\mathfrak{R}}$ and $\boldsymbol{\mu}_{2 \mathfrak{J}}=\boldsymbol{\mu}_{\mathfrak{I}}$, say. The resulting block-diagonal form for the matrix in (31) leads to a similar form for the inverse, and the first relation in (31) reduces to the uncoupled form

$$
\mathbb{Y}=\mathfrak{M}: \mathbb{X}=\mu_{\mathfrak{R}}: \mathbf{X}_{\mathfrak{R}}+\imath \boldsymbol{\mu}_{\mathfrak{I}}: \mathbf{X}_{\mathfrak{J}}
$$

with corresponding simplification of (32).

Replacing $\mathrm{D}$ by $\mathrm{T}=\boldsymbol{\Sigma}+\imath \mathbf{M}$ and $\boldsymbol{\mu}$ by $\boldsymbol{\zeta}$ in (32), one obtains an explicit expression for the Cosserat version of the dissipative yield condition (5):

$$
Y=\|\mathrm{T}\|_{3}^{2}=\mathfrak{R}\left(\mathrm{T}^{*}: 3: \mathrm{T}\right)=1 \text {, with } 3=\mathfrak{K}_{\mathbf{P}}^{*}: \mathfrak{K}_{\mathbf{P}}, \mathfrak{K}_{\mathbf{P}}=\mathfrak{M}_{\mathbf{P}}^{-1},
$$

Special cases of this appear in several past works $[6,44,9]$, with some of the prior history given by Tejchman [2, Chapt. 3]. Although most of those special cases are based on specific constitutive models, they appear to represent variants on the general isotropic form inferred from (45) and cited below in Appendix B.

\section{Hypoplasticity}

With the above definitions, the evolution of stress in Cosserat hypoplasticity is given simply by

$$
\stackrel{\circ}{\mathrm{T}}=\mathfrak{P}(\mathcal{X}, \hat{\mathrm{D}}): \mathbb{D} \text {, with } \mathcal{X}=\{\mathrm{T}, \mathcal{A}\}, \hat{\mathbb{D}}=\mathbb{D} /|\mathbb{D}| \text { and }|\mathbb{D}|=\left(|\mathbf{\Lambda}|^{2}+\xi^{2}|\mathbf{K}|^{2}\right)^{1 / 2}
$$

\footnotetext{
11 The hybrid formulation employed here may nevertheless be useful for purposes of numerical computation, given the ability of current computer software to handle complex arithmetic.
} 
where $\mathfrak{P}$ is defined as in (31) in terms of two complex moduli $\pi_{i}=\boldsymbol{\pi}_{i \mathfrak{R}}+\imath \boldsymbol{\pi}_{i \mathfrak{J}}, i=1,2$, depending on $\mathcal{X}, \hat{\mathbb{D}}$. Here, we have chosen the simplest possible norm $|\mathbb{D}|$ involving a material length scale $\xi$ referred to above, which we recall is associated with a representative particle diameter by other workers $[7,8,21]$. With the replacement of $\mathbf{D}$ by $\mathbb{D}$ in (11), (13) and (14) one obtains the complete parametric Cosserat hypoplasticity, for which some special cases are worth noting.

Whenever $\mathfrak{P}$ in (36) is independent of $\hat{\mathrm{D}}$, we recover Cosserat hypoelasticity, and whenever it is constant, independent of $\{X, \hat{D}\}$ we obtain a concise representation of linear Cosserat elasticity, as formulated e.g. by Mindlin [28, 29], simply by replacing stress rate by stress and deformation rate by infinitesimal strain. In this special case, the symmetric positive-definite form of (32) represents strain energy.

With the goal of achieving thermodynamically admissible forms, one can employ the following extended version of the incremental-elastoplastic form (6):

$$
\mathfrak{P}=\mathfrak{M}_{\mathrm{E}}-\beta\left(\mathfrak{M}_{\mathrm{E}}: \Omega_{\mathrm{P}}: \mathbb{T}\right) \otimes \hat{\mathbb{D}}
$$

with positive-definite elastic modulus $\mathfrak{M}_{\mathrm{E}}$ and plastic compliance $\mathfrak{K}_{\mathrm{P}}$, and with yield condition of the form (35). In a similar way, the present formulation makes for a rather straight-forward extension of (8) to account for dilatancy.

For the isotropic case, Appendix B gives the general form of (36), with a more concrete form of (37), the latter of which involves a total of twelve functions representing various elasticities and compliances. At the time of this writing, the author has not attempted a detailed comparison of the latter against various empiricisms [12, 8, 9, 21, 2].

In closing, it should be noted that rate effects can be included in the above models, simply by allowing various moduli to depend on a non-dimensional variable such as $\tau|\mathbf{D}|$, where $\tau$ denotes a material time-scale, or by a more general viscoplastic model with viscous stresses of the form (1) added to plastic stresses of the type (5) [1].

\section{CONCLUSIONS}

The Abstract and Introduction represent an adequate summary of the present paper. It is hoped that this largely expository work will serve, among other things, to clarify the connections between hypoplasticity, polar as well as non-polar, and more traditional models of elastoplasticity, both of which are currently employed in the mechanics of granular materials. While explicit representations are given here for isotropic models, it would be useful to work out some of the necessary modifications to account for anisotropy based on evolutionary fabric or texture.

\section{ACKNOWLEDGMENTS}

I wish to acknowledge the support of the U.S. National Science Foundation and the influential ideas of the late Professor Ioannis Vardoulakis, particularly his visionary applications of structured continuum models to geomechanics.

\section{REFERENCES}

1. J. Goddard, Granular Mat., accepted and to appear (2009).

2. J. Tejchman, Shear Localization in Granular Bodies with Micro-Polar Hypoplasticity, Geomechanics and Geoengineering, Springer, Berlin; Heidelberg, 2008.

3. D. Kolymbas, Introduction to Hypoplasticity, A.A. Balkema, Rotterdam; Brookfield, 2000.

4. H. B. Mühlhaus, Ingenieur Arch. 56, 389-99 (1986).

5. H. Mühlhaus, and I. Vardoulakis, Géotechnique 37, 271-83 (1987).

6. J. Tejchman, and W. Wu, Acta Mech. 99, 61-74 (1993).

7. J. Tejchman, and E. Bauer, Comput. Geotech. 19, 221-44 (1996).

8. W. Huang, K. Nubel, and E. Bauer, Mech. Mat. 34, 563-76 (2002).

9. S. Mohan, K. Rao, and P. R. Nott, J. Fluid Mech. 457, 377-409 (2002).

10. F. Froiio, G. Tomassetti, and I. Vardoulakis, Int. J. Solids Struct. 43, 7684-720 (2006).

11. P. Nott, Acta Mech. 205, 151-60 (2009).

12. E. Bauer, Mech. Cohes.-Frict. Mater. 5, 125-48 (2000).

13. C. Truesdell, and R. A. Toupin, "Principles of classical mechanics and field theory," in Handbuch der Physik, edited by S. Flügge, Springer, Berlin, 1960, vol. 3/1.

14. C. Truesdell, and W. Noll, The non-linear field theories of mechanics, Encyclopedia of Physics,v. III/3, Berlin ; New York : Springer-Verlag, 1965.

15. G. Gudehus, Soils Found. (J. Jp. Geo. Soc.) 36, 1-12 (1996). 
16. J. D. Goddard, Adv. Coll. Interface Sci. 17, 241-62 (1982).

17. A. C. Pipkin, and R. S. Rivlin, ZAMP 16, 313-26 (1965).

18. G. Gudehus, Powder Tech. 3, 344-51 (1969).

19. D. Kolymbas, Arch. Appl. Mech. 61, 143-51 (1991).

20. W. Wu, E. Bauer, and D. Kolymbas, Mech. Mater. 23, 45-69 (1996).

21. W. Huang, and E. Bauer, Int. J. Num. Anal. Meth. Geomech. 27, 325-52 (2003).

22. E. Bauer, W. Huang, and W. Wu, Int. J. Solids Struct. 41, 5903-19 (2004).

23. R. Hill, Proc. Roy. Soc. A 193, 281-97 (1948).

24. V. C. Prantil, J. T. Jenkins, and P. R. Dawson, J. Mech. Phys. Solids 41, 1357-82 (1993).

25. J. Sun, and S. Sundaresan, "A Plasticity model with microstructure evolution for dense granular flows," in these proceedings, 2009.

26. R. Hill, J. Mech. Phys. Solids 35, 23-33 (1987).

27. J. Ericksen, and C. Truesdell, Arch. Rat. Mech. Anal. 1, 295-323 (1957).

28. R. D. Mindlin, Arch. Ratl. Mech. Anal. 16, 51-78 (1964).

29. S. C. Cowin, Int. J. Solids Struct. 6, 389-98 (1970).

30. C. B. Kafadar, and A. C. Eringen, Int. J. Eng. Sci. 9, 271-305 (1971).

31. J. D. Goddard, "From Granular Matter to Generalized Continuum," in Mathematical models of granular matter, edited by P. Mariano, G. Capriz, and P. Giovine, Springer, Berlin, 2008, vol. 1937 of Lecture Notes in Mathematics, pp. 1-20.

32. P. Giovine, "An Extended Continuum Theory for Granular Media," in Mathematical models of granular matter, edited by P. Mariano, G. Capriz, and P. Giovine, Springer, Berlin, 2008, vol. 1937 of Lecture Notes in Mathematics, pp. 167-92.

33. K.-C. Chen, J.-Y. Lan, and Y.-C. Tai, Int. J. Solids Struct. 46 (2009).

34. W. Pietraszkiewicz, and V. A. Eremeyev, Int. J. Solids Struct. 46, 774-87,2477-80 (2009).

35. M. M. Mehrabadi, S. C. Cowin, and J. Jaric, Int. J. Solids Struct. 32, 439-49 (1995).

36. J. Park, and W. K. Chung, IEEE Trans. Robotics 21, 850-63 (2005).

37. A. Iserles, H. Z. Munthe-Kaas, S. P. Nørsett, and A. Zanna, Acta num. 9, 215-365 (2000).

38. W. Spring, K, Mech. Machine Theo. 21, 365-73 (1986).

39. O. A. Bauchau, and J.-Y. Choi, Nonlin. Dyn. 33, 165-88 (2003).

40. J. Gallier, and D. Xu, Int. J. Robot. Autom. 17, 1-11 (2002).

41. H. Xiao, O. T. Bruhns, and A. Meyers, Acta Mech. 168, 21-33 (2004).

42. M. D. Shuster, J. Aero. Sci. 41, 439-517 (1993).

43. J. D. Goddard, "A note on the representation of Cosserat rotation," in Continuous Media with Microstructure, edited by B. Albers, Springer, Berlin, 2010, vol. to appear.

44. H. Lippmann, Appl. Mech. Revs. 48, 753-62 (1995).

\section{APPENDICES}

\section{A. Plastic potentials}

Considering first the case of non-polar plasticity, we note that, if there exists a plastic potential ${ }^{12} \psi(\mathbf{T})$, then it must be connected to a plastic compliance $\boldsymbol{\kappa}$ like that in (5) by

$$
\hat{\mathbf{D}}=\partial_{\mathbf{T}} \psi /\left|\partial_{\mathbf{T}} \psi\right|=\boldsymbol{\kappa}: \mathbf{T}
$$

The second equality is guaranteed by the existence of a (Legendre) complementary potential $\varphi$, provisionally regarded as a function of $\hat{\mathbf{D}}$, such that $\psi=\mathbf{T}: \partial_{\mathbf{T}} \varphi-\varphi$. However, whenever this relation is invertible, then we may regard $\varphi$ as a function of $\mathbf{T}$, so that $\partial_{\mathbf{T}} \psi=\mathbf{T}: \partial_{\mathbf{T}} \partial_{\mathbf{T}} \varphi$, and (38) is satisfied by taking

$$
\boldsymbol{\kappa}=\frac{\partial_{\mathbf{T}} \partial_{\mathbf{T}} \varphi}{\left|\mathbf{T}: \partial_{\mathbf{T}} \partial_{\mathbf{T}} \varphi\right|}
$$

For example, in the case of an anisotropic (von Mises-Hill) potential [23, 26]:

$$
\psi=\frac{1}{2} \mathbf{T}: \boldsymbol{\psi}: \mathbf{T}=\frac{1}{2} T_{i j} \psi^{i j k l} T_{k l} \text {, with } \psi=\psi^{*} \text { constant, } \Rightarrow \varphi \equiv \psi=\frac{1}{2} \hat{\mathbf{D}}: \boldsymbol{\phi}: \hat{\mathbf{D}}=\frac{1}{2} \mathbf{T}: \boldsymbol{\psi}: \mathbf{T} \text {, with } \boldsymbol{\phi}=\boldsymbol{\psi}^{-1},
$$

and it is easy to show that $\boldsymbol{\kappa}=\boldsymbol{\psi}$ and, hence, that $\boldsymbol{\mu}=\boldsymbol{\kappa}^{-1}=\boldsymbol{\phi}$. The positivity of $\boldsymbol{\kappa}$ obviously implies the convexity of $\varphi$ regarded as function of $\mathbf{T}$.

\footnotetext{
12 The scalar $\psi(\mathbf{T})$ must of course be given as a function of an appropriate set of joint isotropic scalar invariants of $\mathbf{T}$ and a set of tensors sufficient to define the anisotropy. For the Cosserat extension, the joint invariants of $\mathbf{T}$ are replaced by those of $\boldsymbol{\Sigma}, \mathbf{M}$.
} 
The case of Cosserat plasticity is formally covered simply by replacing $\mathbf{D}$ by $\mathrm{D}$ and $\mathbf{T}$ by $\mathrm{T}$ in (38), with

$$
\partial_{\mathbb{T}}=\partial_{\Sigma}-\imath \partial_{\mathbf{M}}
$$

and with suitable modifications of (39), which are not recorded here.

\section{B. Isotropic forms}

With a slight change of notation, the expression of Cowin [29] for the isotropic form of $\mathbf{Y}=\boldsymbol{\mu}: \mathbf{X}$ given by Mindlin [28] can be written

$$
\mathbf{Y}=(\mu+\tau) \mathbf{X}+(\mu-\tau) \mathbf{X}^{\mathrm{T}}+\lambda \operatorname{tr}(\mathbf{X}) \mathbf{I}, \text { i.e. } \mu_{i j k l}=(\mu+\tau) \delta_{i k} \delta_{j l}+(\mu-\tau) \delta_{i l} \delta_{j k}+\lambda \delta_{i j} \delta_{k l},
$$

from which it follows that

$$
\operatorname{Sym} \mathbf{Y}^{\prime}=2 \mu \operatorname{Sym} \mathbf{X}^{\prime}, \operatorname{Skw} \mathbf{Y}=2 \tau \operatorname{Skw} \mathbf{X}, \operatorname{tr}(\mathbf{Y})=\left(\frac{2}{3} \mu+\lambda\right) \operatorname{tr}(\mathbf{X})
$$

representing the orthogonal decomposition into symmetric, skew and spherical parts. The same orthogonality gives the normal quadratic form

$$
\mathbf{X}^{\mathrm{T}}: \boldsymbol{\mu}: \mathbf{X}=2 \mu\left|\operatorname{Sym} \mathbf{X}^{\prime}\right|^{2}+2 \tau|\operatorname{Skw} \mathbf{X}|^{2}+\left(\frac{2}{3} \kappa+v\right)|\operatorname{tr}(\mathbf{X})|^{2},
$$

and the positivity of $\boldsymbol{\mu}$ obviously requires the positivity of $\mu, \tau, \frac{2}{3} \mu+\lambda$. Such positivity is required of strain energy when $\boldsymbol{\mu}$ represents an elastic modulus, or for dissipation when it represents a viscous or plastic modulus.

Also, the inverse form $\mathbf{Y}=\boldsymbol{\kappa}: \mathbf{X}$ follows immediately from (42)-(43) by means of the substitutions:

$$
\mathbf{X} \leftrightarrow \mathbf{Y}, \boldsymbol{\mu} \rightarrow \boldsymbol{\kappa}, \text { with }\{\mu, \tau, \lambda\} \rightarrow\{\kappa, \sigma, \nu\}
$$

and (43) readily gives scalar compliances $\kappa, \sigma, v$ in terms of moduli $\mu, \tau, \lambda$, and vice versa. Without recording these results, we note that self-adjointness, $\boldsymbol{\mu}^{*}=\boldsymbol{\mu}$ and, hence, $\boldsymbol{\kappa}^{*}=\boldsymbol{\kappa}$, is also evident from (42).

Hence, another normal quadratic form

$$
\mathbf{X}^{\mathrm{T}}: \boldsymbol{\zeta}: \mathbf{X}=4 \kappa^{2}\left|\operatorname{Sym} \mathbf{X}^{\prime}\right|^{2}+4 \sigma^{2}|\operatorname{Skw} \mathbf{X}|^{2}+\left(\frac{2}{3} \kappa+v\right)^{2}|\operatorname{tr}(\mathbf{X})|^{2}, \text { where } \boldsymbol{\zeta}=\boldsymbol{\kappa}^{*}: \boldsymbol{\kappa}=\boldsymbol{\kappa}^{2} \stackrel{\text { def }}{=} \boldsymbol{\kappa}: \boldsymbol{\kappa}
$$

follows immediately upon two-fold application of the compliance-form of (43) to calculate $\boldsymbol{\kappa}^{2}$. It is further evident from the now diagonal matrix representation in (31) that

$$
3=\mathfrak{R}^{*}: \mathfrak{R}=\mathfrak{R}^{2}=\boldsymbol{\kappa}_{\mathfrak{R}}^{2}+\imath \boldsymbol{\kappa}_{\mathfrak{I}}^{2} \text {, hence } \mathfrak{R}\left(\mathbb{X}^{*}: 3: \mathbb{X}\right)=\mathbf{X}_{\mathfrak{R}}^{\mathrm{T}}: \boldsymbol{\kappa}_{\mathfrak{R}}^{2}: \mathbf{X}_{\mathfrak{R}}+\mathbf{X}_{\mathfrak{J}}^{\mathrm{T}}: \boldsymbol{\kappa}_{\mathfrak{I}}^{2}: \mathbf{X}_{\mathfrak{I}}
$$

Therefore, the isotropic form of (35) is given simply as the sum of two terms of the form (45), the first obtained by taking $\mathbf{X}=\mathbf{X}_{\mathfrak{R}}=\boldsymbol{\Sigma}, \boldsymbol{\kappa}=\boldsymbol{\kappa}_{\mathfrak{R}}$, and the second by taking $\mathbf{X}=\mathbf{X}_{\mathfrak{J}}=\mathbf{M}, \boldsymbol{\kappa}=\boldsymbol{\kappa}_{\mathfrak{J}}$ with $\kappa, \sigma, v$ replaced, respectively, by rotational compliances, say, $\breve{\kappa}, \breve{\sigma}, \breve{v}$.

Furthermore, with $\boldsymbol{\mu}(\mu, \tau, \lambda)$ defined in (42), we immediately obtain the linear isotropic form of (36) by means of (34) and (36) :

$$
\stackrel{\circ}{\Sigma}=\pi_{\mathfrak{R}}: \Lambda, \text { and } \stackrel{\circ}{\mathbf{M}}=\boldsymbol{\pi}_{\mathfrak{J}}: \mathbf{K}
$$

with

$$
\boldsymbol{\pi}_{\mathfrak{R}}=\boldsymbol{\mu}(\mu, \tau, \lambda) \text { and } \boldsymbol{\pi}_{\mathfrak{I}}=\boldsymbol{\mu}(\breve{\mu}, \breve{\tau}, \breve{\lambda})
$$

which are uncoupled, linear versions of (9), involving six elasticities ${ }^{13} \mu, \lambda, \tau, \breve{\mu}, \breve{\tau}, \breve{\lambda}$.

The nonlinear isotropic version of parametric Cosserat hypoplasticity is also given by (48), with moduli $\boldsymbol{\pi}_{\mathfrak{R}}, \boldsymbol{\pi}_{\mathfrak{I}}$ depending on $\mathbb{T}, \mathcal{A}, \hat{\mathrm{D}}$, where $\mathcal{A}$ represents a set of evolutionary scalar parameters. The resulting equations are now generally coupled, because of their joint dependence on $\mathrm{T}=\boldsymbol{\Sigma}+\imath \mathbf{M}, \mathcal{A}, \hat{\mathrm{D}}=\mathbf{\Lambda}+\imath \mathbf{K}$.

If one further adopts the incremental elastoplastic form (6), then each of the moduli $\boldsymbol{\pi}_{\mathfrak{R}}, \boldsymbol{\pi}_{\mathfrak{J}}$ is defined by its own isotropic elastic modulus $\boldsymbol{\mu}_{\mathrm{E}}$ and plastic compliance $\boldsymbol{\kappa}_{\mathrm{P}}$, of the type defined above, which in turn are given by a total of twelve elastic moduli and plastic compliances $\mu_{\mathbf{E}}, \ldots, v_{\mathbf{P}}$ and $\breve{\mu}_{\mathbf{E}}, \ldots, \breve{v}_{\mathbf{P}}$. The latter must in general be treated as functions of evolutionary scalar parameters $\mathcal{A}$ and the joint isotropic scalar invariants of $\mathbb{T}, \mathbb{D}$.

\footnotetext{
13 Which are easily related to Cowin's [29] moduli $\mu, \lambda, \tau, \eta, \eta^{\prime}, \alpha$. The notation employed here leads to appreciably simpler conditions for the positivity of quadratic forms such as (44), with conditions on $\breve{\mu}, \breve{\tau}, \breve{\lambda}$ identical with those on $\mu, \tau, \lambda$.
} 